\title{
Diacronie
}

Studi di Storia Contemporanea

$N^{\circ} 23,3$ | 2015

Mediterraneo cosmopolita

\section{Bernardino Osio (a cura di), Lettere da Costantinopoli (1914-1915). Carteggio familiare di Bernardino Nogara}

\section{Matteo Anastasi}

\section{(2) OpenEdition}

Journals

Edizione digitale

URL: http://journals.openedition.org/diacronie/2397

DOI: $10.4000 /$ diacronie.2397

ISSN: 2038-0925

Editore

Association culturelle Diacronie

Notizia bibliografica digitale

Matteo Anastasi, «Bernardino Osio (a cura di), Lettere da Costantinopoli (1914-1915). Carteggio familiare di Bernardino Nogara », Diacronie [Online], $N^{\circ}$ 23, 3 | 2015, documento 4, Messo online il 29 octobre 2015, consultato il 24 septembre 2020. URL : http://journals.openedition.org/diacronie/2397 ; DOI : https://doi.org/10.4000/diacronie.2397 


\title{
Diacronie
}

N. 23 | 3|2015 Mediterraneo cosmopolita: le relazioni culturali tra Turchia ed Europa

\section{RECENSIONE:}

\section{Bernardino OSIO (a cura di), Lettere da} Costantinopoli (1914-1915). Carteggio familiare di Bernardino Nogara, Firenze, Centro Di, 2014, 174 pp.

\author{
a cura di Matteo ANASTASI *
}

Diplomatico di carriera per quasi cinquant'anni (in servizio dal 1960 al 2008), l'ambasciatore milanese Bernardino Osio ha operato in Svizzera, Argentina, presso la Santa Sede, in Ecuador, Spagna e Perù. Da ultimo è stato Segretario Generale dell'Istituto Italo-Latinoamericano e, a Parigi, dell'Unione Latina. Fine scrittore, Osio ha pubblicato diverse opere. Oltre a due interessanti testi memorialistici ${ }^{1}$, nel 2006 si è occupato della figura di Cesare Cantù, lavorando su alcune epistole private del patriota lombardo ${ }^{2}$.

In Lettere da Costantinopoli la penna di Osio prende ancora le mosse da un archivio domestico. Stavolta il suo. Attingendo infatti dalla ricchissima documentazione familiare, Osio raccoglie e commenta una serie di lettere che il nonno materno Bernardino Nogara (1870-1958) indirizza dalla capitale ottomana alla moglie Ester, rientrata in Italia con i cinque figli nel giugno del 1914, alla vigilia dello scoppio della Grande Guerra.

Ingegnere, imprenditore, diplomatico e finanziere, Nogara è stato uno degli uomini più importanti nella storia d'Italia del primo Novecento. Residente fin dal 1907 a Costantinopoli in qualità di direttore della Società Commerciale d'Oriente (COMOR), nel 1911 è incaricato dal quarto governo di Giovanni Giolitti - con il marchese Antonino di San Giuliano agli Esteri - di condurre a Costantinopoli le trattative che pongono fine

${ }_{1}^{1}$ OSIO, Bernardino, Ande e monasteri. Tre anni in Ecuador 1981-1984, Firenze, Centro Di, 2010; ID., Calagrande, ovvero degli Hortuli Hosiani. Breve storia a confronto degli amanti nipoti, Firenze, Centro Di, 2011.

2 ID., Dalle lettere familiari di Cesare Cantù. Una gita sul Lario per il Corpus Domini del 187o, Milano, Cisalpino, 2006 
alla guerra italo-turca. Un lavoro positivamente svolto che confluisce nel trattato di Ouchy dell'ottobre 1912. L'abilità dimostrata in quella circostanza vale a Nogara la nomina a delegato italiano al Debito Pubblico Ottomano. È quindi sempre da Costantinopoli che muove il carteggio oggetto della pubblicazione. Il periodo preso in esame da Osio si staglia sull'arco di un anno, comprendendo la fase che va dal 2 luglio 1914 all'11 luglio 1915. Si tratta di una corrispondenza che, dopo lo scoppio della guerra, è resa difficoltosa dall’interruzione di molteplici vie di comunicazione e da una censura man mano sempre più pressante. Ogni missiva contiene notizie di carattere privato accompagnate da ampie considerazioni sugli sviluppi della politica internazionale e sulle prospettive che emergono per l'Italia alla vigilia del primo conflitto mondiale. Sotto quest'ultimo aspetto Nogara dimostra una notevole stima intellettuale per la consorte: con lei condivide riflessioni e analisi, inviandole a più riprese documenti ufficiali (es. il libro bianco inglese sulle origini della guerra, chiedendole sue considerazioni in merito). Da quell'inchiostro emergono chiare le idee di Nogara. Mentre gli Imperi tedesco e austro-ungarico premono per l'ingresso nelle ostilità di Bulgaria e Turchia, egli osserva - assai correttamente dati gli sviluppi successivi - che, se Costantinopoli si fosse lasciata coinvolgere nel conflitto, l'Impero ottomano sarebbe crollato e avrebbe visto l'Europa gettarsi sulle sue spoglie3. Timori che iniziano a rivelarsi fondati alla fine di luglio del 1914, quando gli incrociatori tedeschi Goeben e Breslau fanno il loro ingresso nel Mar di Marmara. In questo clima, che rischia di accelerare l'entrata della Sublime Porta in guerra, Nogara ha il difficile compito di garantire il rispetto del Debito Pubblico Ottomano, nel cui consiglio rappresenta gli interessi italiani e che costituisce la sua principale fonte di sostentamento personale.

Quanto allo stallo di Roma, Nogara si lascia andare a considerazioni critiche. «In quest'epoca guerresca, durante la quale si decide l'avvenire dell'Europa, desiderare che l'Italia rimanga sempre coll'arma al piede» ${ }^{4}$ gli sembra privo di senso: «Gli assenti hanno sempre torto e l'Italia perderebbe immediatamente il rango di grande potenza»5. Da queste riflessioni emerge dunque un Nogara interventista e, altresì, favorevole al riorientamento delle alleanze che avrebbe portato Roma al fianco dell'Intesa. A suo parere, le sorti del conflitto sono nelle mani della Gran Bretagna e a Londra occorre guardare per trarre il massimo dalle dinamiche postbelliche ${ }^{6}$. Nogara auspica, tuttavia, temendo anche per la sua situazione personale, che il governo Giolitti non opti per uno

3 Cfr. OSIO, Bernardino (a cura di), Lettere da Costantinopoli (1914-1915). Carteggio familiare di Bernardino Nogara, Firenze, Centro Di, 2014, p. 52.

4 Ibidem, p. 70.

5 Ibidem, pp. 70-71.

${ }^{6}$ Ibidem, p. 56. 
scontro frontale con gli ottomani, lasciandosi «prendere dal gusto di venire a infrangersi contro i Dardanelli»7.

Nel frattempo si sviluppano due importanti dinamiche di esclusione. Da un lato la città si svuota dell'elemento latino che lascia spazio al carattere teutonico: il governo fa cancellare dalle attività commerciali le insegne con caratteri latini mentre concede ai tedeschi il permesso di utilizzare quelle con caratteri gotici. Dall'altro lato, le minoranze armene e greche sono vittime di emigrazioni forzate. Gli Armeni, residenti sulle coste del Mar Nero, sono costretti a riparare in Mesopotamia preludendo quello che passerà alla storia come il secondo massacro etnico perpetrato ai loro danni. I greci, installati nella zona del Mar di Marmara, vengono internati e costretti a pane e acqua: Nogara scrive di oltre quarantamila sfollati di etnia ellenica.

Sul finire del 1914, l'ingresso ottomano in guerra coincide, inoltre, con un consistente rallentamento dell'attività finanziarie presiedute da Nogara. Fin dal mese di ottobre le difficoltà economiche della COMOR lo costringono a numerosi licenziamenti e alla diminuzione degli stipendi dei dipendenti superstiti. Una situazione assai complicata, umanamente e professionalmente, che Nogara deve gestire da solo. Il complicarsi dei collegamenti con Roma, infatti, impedisce le comunicazioni. A più riprese scrive per conoscere il da farsi. L'ultimo vano tentativo è datato giugno $1915 \mathrm{ma}$ oramai, con l'Italia ufficialmente in guerra, non giungono più indicazioni neanche dalla Camera di Commercio ${ }^{8}$. Riesce, tuttavia, rocambolescamente, a mettersi in contatto con Roma all’inizio di luglio. Ottiene libertà d'azione, "più per partire che per restare» 9 , e decide di scegliere la prima opzione. Lascia Costantinopoli il 18 luglio 1915, indirizzando l'ultimo pensiero ai suoi «protetti che rimarranno molto più in balia degli eventi» ${ }^{10}$.

Il volume preso in esame, fondandosi sulla corposa documentazione inedita studiata da Osio, costituisce un'opera preziosa per diverse ragioni. Anzitutto tocca spazi e cruciali della storia italiana del XX secolo: un anno - la fase giugno 1914-luglio 1915 dove il lavoro della Consulta e di tutte le figure che guardano ad essa (come Nogara) è rovente e culmina con la scelta di entrare in guerra.

Inoltre, e questo è un grande merito, permette di fare luce su un profilo di grande spessore, quale quello di Bernardino Nogara, del quale ancora non è stata pubblicata

\footnotetext{
7 Ibidem, p. 62.

8 Ancora il 4 luglio scrive: «Ed io parto, oppure non parto: come non ho tue notizie, così non ho notizie della Camera di Commercio di Roma, e non so quali pesci pigliare: se si parte da qui, più non si ritorna, se non a guerra finita, e la guerra potrebbe durare ancora un anno. Con questa propsettiva è prudente prendere abbandonare il posto? Bisogna mettere a dormire il cuore per prendere una decisione». Ibidem, p. 164.

9 Ibidem, p. 165.

${ }^{10}$ Ibidem.
} 
una biografia esaustiva ${ }^{11}$. Più in generale, permette di esaminare con rinnovato slancio il ruolo di quei tecnici che, come Nogara e l'amico Giuseppe Volpi, lavorano all'estero nel primo Novecento per fare gli interessi del Paese, gettando le basi per importanti prospettive economiche, imprenditoriali e tecnologiche.

Nondimeno, l'opera fornisce un interessantissimo spaccato sulle relazioni personali italo-turche prima e durante il conflitto. Curiosità e sospetto si fondono nelle descrizioni di Nogara, fra le quali spiccano, in particolare, quella di Enver Pascià, uno dei triumviri del Comitato Unione e Progresso e ministro della Guerra; di Halil Bey, vicepresidente della Camera dei Deputati e poi ministro degli Esteri; di Reof Bey, ministro della Marina; di Nizami Osman, ministro dei Lavori Pubblici.

11 A oggi l'unico tentativo biografico è rintracciatabile in una tesi di dottorato discussa all'Università di Firenze dal dott. Angelo Caleca sotto la supervisione dei proff. Luca Mannori e Luciano Segreto. Cfr. CALECA, Angelo, Al servizio dell'Italia e del Papa. Le tante vite di Bernardino Nogara (1870-1958), tesi di dottorato in Storia Economica, Università degli Studi di Firenze, Firenze, a.a. 2011/2012. 


\section{* L'autore}

Matteo Anastasi ha conseguito la laurea in Storia presso l'Università Europea di Roma discutendo una tesi sull'attività del quarto governo di Amintore Fanfani. Attualmente si sta specializzando in Relazioni Internazionali presso l'Università LUISS Guido Carli, sotto la supervisione del professor Francesco Perfetti. I suoi campi di studio sono l'analisi del fenomeno politico dell' apertura a sinistra nel dopoguerra e la storia diplomatica italiana.

URL: < http://www.studistorici.com/progett/autori/\#Anastasi >

\section{Per citare questo articolo:}

ANASTASI, Matteo, «Recensione: Bernardino OSIO (a cura di), Lettere da Costantinopoli (1914-1915). Carteggio familiare di Bernardino Nogara, Firenze, Centro Di, 2014, 174 pp.», Diacronie. Studi di Storia Contemporanea : Mediterraneo cosmopolita: le relazioni culturali tra Turchia ed Europa, 29/10/2015,

URL:< http://www.studistorici.com/2015/10/29/anastasi_numero_23/ >

Diacronie Studi di Storia Contemporanea $\}$ www.diacronie.it

Risorsa digitale indipendente a carattere storiografico. Uscita trimestrale.

redazione.diacronie@hotmail.it

Comitato di redazione: Jacopo Bassi - Luca Bufarale - Elisa Grandi - Deborah Paci - Fausto Pietrancosta - Matteo Tomasoni - Luca Zuccolo

Diritti: gli articoli di Diacronie. Studi di Storia Contemporanea sono pubblicati sotto licenza Creative Commons 2.5 Possono essere riprodotti a patto di non modificarne i contenuti e di non usarli per fini commerciali. La citazione di estratti è comunque sempre autorizzata, nei limiti previsti dalla legge. 